\title{
取代黄酩磷酸酯的合成及抗细菌活性研究
}

$\begin{array}{ccrrrr}\text { 黄民国阮祥辉 } & \text { 张菊平 李 } & \text { 琴 } & \text { 王一会 } \\ \text { 陈丽娟 张 } & \text { 橙 李 } & \text { 普 } & \text { 薛 } & \text { 伟* }\end{array}$

(贵州大学精细化工研究开发中心 绿色农药与农业生物工程国家重点实验室培育基地 教育部绿色农药与农业生物 工程重点实验室 贵阳 550025)

\begin{abstract}
摘要 将取代黄酮醇引入亚磷酸二乙酯，设计合成了 14 个含取代黄酮基磷酸酯类新化合物，通过 ${ }^{1} \mathrm{H} N \mathrm{NM},{ }^{13} \mathrm{C} \mathrm{NMR}$, ${ }^{31} \mathrm{P} N M R, M S, I R$ 和 HRMS 对它们的结构进行了表征. 采用浊度法测试了目标化合物的离体抑菌活性, 表明部分目标化 合物在 $100 \mu \mathrm{g} / \mathrm{mL}$ 浓度下对水稻白叶枯病菌表现出良好的抑制率, 明显优于对照药剂噻菌铜和叶枯唑 $(\geqslant 57.4 \%)$. 其中 $O, O^{\prime}$-二乙基- $O^{\prime \prime}$-( $\left(2^{\prime}, 7\right.$-二甲氧基黄酮-3-基)磷酸酯(2b)、 $O, O^{\prime}$-二乙基- $O^{\prime \prime}$-(2'-甲氧基-7-乙氧基黄酮-3-基)磷酸酯(2f) 和 $O, O^{\prime}$ 二乙基- $O^{\prime \prime}$-(3',4'-二甲氧基-7-乙氧基黄酮-3-基)磷酸酯(2m)在 $100 \mu \mathrm{g} / \mathrm{mL}$ 浓度下对水稻白叶枯病菌的抑制率分别达到 92.8\%, 87.7\% 和 88.3\%. $O, O^{\prime}$-二乙基- $O^{\prime \prime}$-(4'-氟-7-乙氧基黄酮-3-基)磷酸酯(2h)和 $O, O^{\prime}$-二乙基- $O^{\prime \prime}$-(4'-甲基-7-乙氧基黄酮3-基)磷酸酯(2k)在 $100 \mu \mathrm{g} / \mathrm{mL}$ 浓度下对柑橘溃疡病菌也有较好的抑制活性，其抑制率分别为 $85.1 \%$ 和 $71.7 \%$.
\end{abstract}

关键词 亚磷酸酯; 黄酮醇; 黄酮磷酸酯衍生物; 抗细菌活性

\section{Synthesis and Antibacterial Activity of Novel Phosphonate Derivatives Containing Flavonoids}

\author{
Huang, Minguo $\quad$ Ruan, Xianghui Zhang, Juping Li, Qin Wang, Yihui \\ Chen, Lijuan Zhang, Cheng Li, Pu Xue, Wei*
}

(State Key Laboratory Breeding Base of Green Pesticide and Agricultural Bioengineering, Key Laboratory of Green Pesticide and Agricultural Bioengineering, Ministry of Education, Center for Research and Development of Fine Chemicals, Guizhou University, Guiyang 550025)

\begin{abstract}
By introducing flavonoids group into H-phosphonate, fourteen novel phosphonate derivatives containing flavonoid unit were designed and synthesized. Their structures were characterized by ${ }^{1} \mathrm{H}$ NMR, ${ }^{13} \mathrm{C}$ NMR, ${ }^{31} \mathrm{P}$ NMR, MS, IR and HRMS, and their antibacterial activities were evaluated via turbidimeter test in vitro. The bioassays results reveal that part of the target compounds exhibit better activities against Xanthomonas oryzae pv. Oryzae (Xoo) and Xanthomonas axonopodis pv. Citri (Xac) than positive controls thiodiazole copper and bismerthiazol $(\geqslant 57.4 \%)$. Among them, the inhibitory rates of (2-(2-methoxyphenyl)-7-methoxy-4-oxo-4H-chromen-3-yl)diethyl phosphonate (2b), (2-(2-methoxyphenyl)-7-ethoxy-4-oxo$4 H$-chromen-3-yl)diethyl phosphonate (2f) and (2-(3,4-dimethoxyphenyl)-7-ethoxy-4-oxo-4H-chromen-3-yl)diethyl phosphonate $(\mathbf{2 m})$ reached $92.8 \%, 87.7 \%$ and $88.3 \%$ against $X o o$ at a concentration of $100 \mu \mathrm{g} / \mathrm{mL}$, respectively. In addition, the inhibitory rates of (2-(4-fluorophenyl)-7-ethoxy-4-oxo-4H-chromen-3-yl)diethyl phosphonate (2h) and (2-( $p$-tolyl)-7-ethoxy-4-oxo$4 H$-chromen-3-yl)diethyl phosphonate $(\mathbf{2 k})$ reached $85.1 \%$ and $71.7 \%$ against $\mathrm{Xac}$ at a concentration of $100 \mu \mathrm{g} / \mathrm{mL}$, respectively.

Keywords H-phosphonate; flavonol; phosphonate derivatives containing flavonoid; antibacterial activity
\end{abstract}

$\mathrm{H}$-亚磷酸酯含有强极性的磷酰基 $(\mathrm{P}=\mathrm{O})$, 是一个重 要的活性基团. 其特殊的结构使其具有化学活性高和反 应位点多等优点, 化合物的反应多样性主要由磷原子和
烷氧基的 $\alpha$-碳原子两个反应中心所决定，涉及 $\mathrm{P}-\mathrm{H}$ 和 $\mathrm{P}=\mathrm{O}$ 官能键. 由于 $\mathrm{H}$-亚磷酸酯兼具廉价易得、易保存、 结构稳定等优点, H-亚磷酸酯及其衍生物作为抗病毒

* Corresponding author. E-mail: wxue@gzu.edu.cn

Received January 14, 2017; revised February 28, 2017; published online April 18, 2017.

Project supported by the National Natural Science Foundation of China (No. 21462012) and the Excellent Young Talents of Science and Technology in Guizhou Province (No. 201535).

国家自然科学基金(No. 21462012)和贵州省优秀青年科技人才(No. 201535)资助项目. 
剂 ${ }^{[1]}$ 、杀菌剂 ${ }^{[2]} 、$ 除草剂 ${ }^{[3]}$ 、植物调节剂 ${ }^{[4]}$ 、食品添加剂 及抗癌药 ${ }^{[5]}$ 等, 被广泛应用于工业、农业以及制药等行 业.

黄酮醇类化合物作为生物体内的次生代谢物广泛 分布于大自然中, 因其具有多种多样的生理活性而备受 国内外重视 ${ }^{[6]}$. 目前, 黄酮醇化合物的生物活性研究集 中在心血管系统 ${ }^{[7]}$ 、病毒 ${ }^{[8]}$ 、炎症 ${ }^{[9]}$ 和肿瘤 ${ }^{[10]}$ 等方面, 关 于黄酮化合物抗菌活性报道仅限于少数天然产物中分 离的黄酩醇类化合物, 关于黄酮类化合物抗菌作用的系 统研究未见报道, 针对农经作物上的常见植物细菌病害 研究更是少见. 众所周知, 植物细菌病害造成的腐烂、 畸形、枯萎、斑点和变色等影响农经作物的正常生长和 品质, 威胁粮食安全. 当前, 化学杀菌剂是防治作物细 菌的主要手段, 且品种比较单一, 防治作物细菌病害的 有效途径有限且效果不理想 ${ }^{[11,12]}$. 拼合原理是设计创新 药物的重要思想, 目前已有许多成功的范例, 鉴于黄酮 醇和亚磷酸酯的生物活性, 本工作拟设计合成以黄酮醇 为结构骨架的磷酸酯类化合物, 研究它们的结构与活性 关系，以期获得较高抑制植物病菌的先导化合物. 作为 一种尝试, 本工作将系列取代黄酮醇分别引入亚磷酸二 乙酯，设计合成了 14 个未见报道的取代黄酮磷酸酯类 化合物 2a $\sim 2 n$, 合成路线见 Scheme 1. 采用浊度法 ${ }^{[13,14]}$ 测试了目标化合物抗植物细菌活性, 发现部分化合物具 有超过阳性对照物的活性, 验证了设计思想, 值得进一 步研究

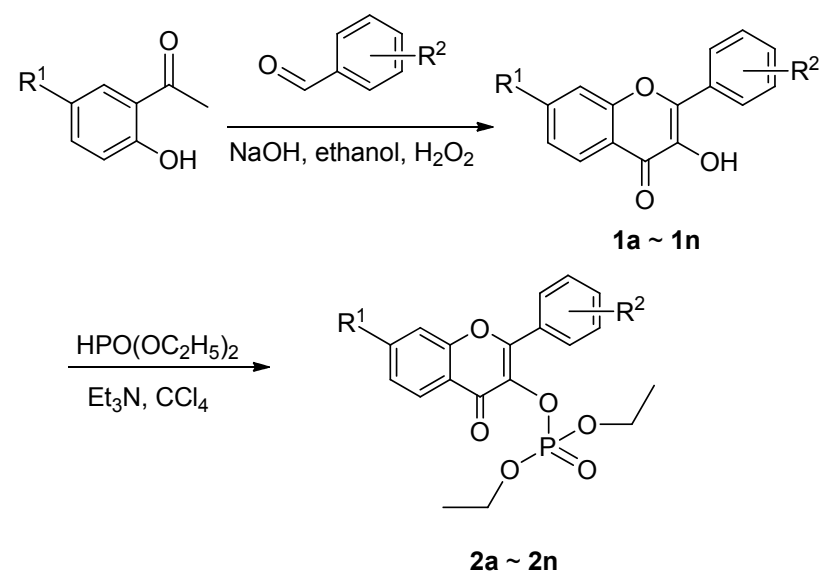

a: $R^{1}=7-\mathrm{OCH}_{3}, \mathrm{R}^{2}=4^{\prime}-\mathrm{OCH}_{3} ; \mathbf{b}: \mathrm{R}^{1}=7-\mathrm{OCH}_{3}, \mathrm{R}^{2}=2^{\prime}-\mathrm{OCH}_{3}$ c: $\mathrm{R}^{1}=7-\mathrm{OCH}_{3}, \mathrm{R}^{2}=4^{\prime}-\mathrm{Cl}$; d: $\mathrm{R}^{1}=7-\mathrm{OCH}_{3}, \mathrm{R}^{2}=4^{\prime}-\mathrm{C}\left(\mathrm{CH}_{3}\right)_{3}$; e: $R^{1}=7-\mathrm{OCH}_{2} \mathrm{CH}_{3}, \mathrm{R}^{2}=4^{\prime}-\mathrm{OCH}_{3} ;$ f: $\mathrm{R}^{1}=7-\mathrm{OCH}_{2} \mathrm{CH}_{3}, \mathrm{R}^{2}=2^{\prime}-\mathrm{OCH}_{3}$ g: $\mathrm{R}^{1}=7-\mathrm{OCH}_{2} \mathrm{CH}_{3}, \mathrm{R}^{2}=2^{\prime}-\mathrm{Br} ; \mathbf{h}: \mathrm{R}^{1}=7-\mathrm{OCH}_{2} \mathrm{CH}_{3}, \mathrm{R}^{2}=4^{\prime}-\mathrm{F}$; i: $R^{1}=7-\mathrm{OCH}_{2} \mathrm{CH}_{3}, \mathrm{R}^{2}=4^{\prime}-\mathrm{Cl}$; j: $\mathrm{R}^{1}=7-\mathrm{OCH}_{2} \mathrm{CH}_{3}, \mathrm{R}^{2}=3^{\prime}-\mathrm{Br}$; k: $\mathrm{R}^{1}=7-\mathrm{OCH}_{2} \mathrm{CH}_{3}, \mathrm{R}^{2}=4^{\prime}-\mathrm{CH}_{3} ;$ I: $\mathrm{R}^{1}=7-\mathrm{OCH}_{2} \mathrm{CH}_{3}, \mathrm{R}^{2}=\mathrm{H}$; m: $\mathrm{R}^{1}=7-\mathrm{OCH}_{2} \mathrm{CH}_{3}, \mathrm{R}^{2}=3^{\prime}-\mathrm{OCH}_{3}-4^{\prime}-\mathrm{OCH}_{3}$; n: $\mathrm{R}^{1}=7-\mathrm{OCH}_{2} \mathrm{CH}_{3}, \mathrm{R}^{2}=2^{\prime}-\mathrm{OCH}_{3}-4^{\prime}-\mathrm{OCH}_{3}$

图式 1 目标化合物 $\mathbf{2 a} \sim 2 \mathrm{n}$ 的合成路线

Scheme 1 Synthetic route of target compounds $\mathbf{2 a} \sim \mathbf{2 n}$

\section{1 结果与讨论}

\section{1 目标化合物的合成及结构表征}

以系列黄酮醇和亚磷酸酯为原料, 三乙胺为催化 剂, 四氯化碳为溶剂，反应 $24 \mathrm{~h}$, 合成系列取代黄酮磷 酸二乙酯化合物. 在本研究中, 若以四氯化碳为溶剂、 氨或其他无机碱作为催化剂时，薄层色谱(TLC)跟踪发 现反应副产物较多或不反应。通过对反应条件的摸索, 我们发现以四氯化碳为溶剂, 三乙胺为催化剂, 最后能 以较高收率得到目标化合物. 相应的反应机理为: 首先 亚磷酸酯与三乙胺反应生成的亚磷酸酯负离子与四氯 化碳反应生成氯代磷酸酯, 最后黄酮醇在三乙胺的催化 下与反应过程中产生的氯代磷酸酯反应得到目标化合 物, 由此可知, 三乙胺作为催化剂更有利于在反应过程 中氯代磷酸酯的产生 ${ }^{[15,16]}$.

在 IR 中, 化合物 $\mathbf{2 a} \sim 2 \mathbf{n}$ 的 $\mathrm{P}=\mathrm{O}$ 的伸缩振动峰在 $1300 \sim 1250 \mathrm{~cm}^{-1}$ 左右，黄酮骨架上酮羰基 $\mathrm{C}=\mathrm{O}$ 的振动 峰在 $1645 \sim 1603 \mathrm{~cm}^{-1}$. 在 ${ }^{1} \mathrm{H}$ NMR 中, 饱和碳上的质子 化学位移位于 $\delta 1.00 \sim 4.50$ 范围内; 在 ${ }^{13} \mathrm{C} N \mathrm{NMR}$ 中, 饱 和碳的化学位移均在高场区, 出现在 $\delta \quad 10.00 \sim 70.00$ 之 间; 在 ${ }^{31} \mathrm{P} N M R$ 中, $\mathbf{2 a} \sim \mathbf{2 n}$ 的 $\mathrm{P}$ 呈现单峰处于 $\delta$ $-4.00 \sim-6.11$ 范围内. 在 ESI-MS 中, $2 \mathbf{a} \sim 2 \mathrm{n}$ 均出现较 强的 $[\mathrm{M}+\mathrm{H}]^{+}$和 $[\mathrm{M}+\mathrm{Na}]^{+}$峰. 在 HRMS 谱图中, 目标产 物的 HRMS $[\mathrm{M}+\mathrm{H}]^{+}$实测值和理论计算值是一致的，误 差均在 $m / z \pm 0.003$ 内，进一步证实了目标化合物的结 构.

\section{2 目标化合物的抗植物病菌活性分析}

目标化合物 $\mathbf{2 a} \sim 2 \mathbf{n}$ 的抗菌活性测试结果见表 1 . 从 该表可知, 部分化合物在 $100 \mu \mathrm{g} / \mathrm{mL}$ 浓度下对水稻白叶 枯病菌表现出优良的抑制活性，明显高于对照药剂噻菌 铜(34.3\%)和叶枯唑(57.4\%). 部分化合物对相橘溃疡病 菌表现出一定的抑菌活性. 其中 $2 \mathbf{h}$ 和 $2 \mathbf{k}$ 在 $100 \mu \mathrm{g} / \mathrm{mL}$ 浓度下对柑橘溃疡病菌的抑制率分别为 $85.1 \%$ 和 $71.7 \%$.

以商品化的叶枯唑为对照药剂, 对化合物 $2 \mathbf{b}, 2 \mathrm{e}$, $2 \mathbf{f}, 2 \mathbf{k}$ 和 $2 \mathrm{~m}$ 进行了毒力回归方程和 $\mathrm{EC}_{50}$ 值测试, 结果 见表 2. 从表 2 可知, 上述 5 个化合物对水稻白叶枯病菌 的抑制率活性明显优于对照药剂叶枯唑. 其中，化合物 $2 \mathrm{f}$ 和 $2 \mathrm{~m}$ 对水稻白叶枯病菌的 $\mathrm{EC}_{50}$ 值分别为 22.78 和 $16.47 \mu \mathrm{g} / \mathrm{mL}$ ，远低于叶枯唑的 $88.51 \mu \mathrm{g} / \mathrm{mL}$.

初步构效关系分析表明，当 $\mathrm{R}^{2}$ 为甲氧基时，不管是 邻位单取代基还是对位单取代基，在 100 和 $50 \mu \mathrm{g} / \mathrm{mL}$ 两个供试浓度下, 化合物 $\mathbf{2 a}, \mathbf{2 b}, \mathbf{2 e}$ 和 $\mathbf{2 f}$ 对水稻白叶枯 病菌的抑制率均较好，进一步分析，取代基 $\mathrm{R}^{2}$ 为 $2^{\prime}-\mathrm{OCH}_{3}$ 的化合物 $\mathbf{2 b}$ 和 $2 \mathbf{f}$ 在 100 和 $50 \mu \mathrm{g} / \mathrm{mL}$ 两个供试 
表 1 目标化合物 $\mathbf{2 a} \sim \mathbf{2 n}$ 的抗细菌活性(抑制率/\% $)^{a}$

Table 1 Antibacterial activities (inhibition rate/\%) of target compounds $\mathbf{2 a} \sim \mathbf{2 n}$

\begin{tabular}{|c|c|c|c|c|}
\hline \multirow{2}{*}{ Compd. } & \multicolumn{2}{|c|}{ Xanthomonas oryzae pv. oryzae } & \multicolumn{2}{|c|}{ Xanthomonas axonopodis pv. citri } \\
\hline & $100 \mu \mathrm{g} / \mathrm{mL}$ & $50 \mu \mathrm{g} / \mathrm{mL}$ & $100 \mu \mathrm{g} / \mathrm{mL}$ & $50 \mu \mathrm{g} / \mathrm{mL}$ \\
\hline $2 \mathbf{2 a}$ & $55.0 \pm 3.8$ & $44.5 \pm 1.2$ & $8.2 \pm 1.4$ & $2.8 \pm 1.1$ \\
\hline $2 \mathbf{b}$ & $92.8 \pm 0.5$ & $58.2 \pm 1.4$ & $40.7 \pm 3.3$ & $38.6 \pm 3.3$ \\
\hline $2 c$ & $49.3 \pm 4.4$ & $12.8 \pm 3.0$ & $42.6 \pm 2.1$ & $22.9 \pm 1.6$ \\
\hline 2d & $49.3 \pm 2.2$ & $45.5 \pm 1.4$ & $37.1 \pm 2.7$ & $34.6 \pm 1.0$ \\
\hline $2 e$ & $61.4 \pm 3.7$ & $56.6 \pm 1.6$ & $23.4 \pm 1.8$ & $20.3 \pm 2.6$ \\
\hline $2 f$ & $87.7 \pm 1.1$ & $69.2 \pm 2.6$ & $42.3 \pm 2.7$ & $32.7 \pm 2.7$ \\
\hline $2 \mathrm{~g}$ & $14.4 \pm 2.0$ & $12.7 \pm 3.2$ & $44.5 \pm 3.1$ & $40.9 \pm 1.8$ \\
\hline $2 \mathrm{~h}$ & $17.3 \pm 2.6$ & $7.7 \pm 2.7$ & $85.1 \pm 3.3$ & $65.7 \pm 2.9$ \\
\hline $2 \mathbf{i}$ & $44.3 \pm 1.4$ & $22.8 \pm 2.1$ & $60.7 \pm 1.9$ & $41.3 \pm 2.8$ \\
\hline $2 \mathbf{j}$ & $51.3 \pm 3.0$ & $37.0 \pm 2.6$ & $36.6 \pm 2.6$ & $23.2 \pm 2.9$ \\
\hline $2 \mathbf{k}$ & $74.4 \pm 2.0$ & $53.5 \pm 3.9$ & $71.7 \pm 2.1$ & $48.9 \pm 1.7$ \\
\hline 2I & $55.8 \pm 2.0$ & $28.5 \pm 1.9$ & $33.3 \pm 2.6$ & $28.2 \pm 3.9$ \\
\hline $2 m$ & $88.3 \pm 1.7$ & $64.1 \pm 3.3$ & $10.1 \pm 2.9$ & $7.1 \pm 1.7$ \\
\hline $2 n$ & $35.7 \pm 3.1$ & $26.1 \pm 2.1$ & $43.3 \pm 1.4$ & $36.5 \pm 1.2$ \\
\hline Thiodiazole-copper $^{b}$ & $34.3 \pm 2.2$ & $20.8 \pm 2.1$ & $20.2 \pm 3.4$ & $10.9 \pm 2.5$ \\
\hline Bismerthiazol $^{b}$ & $57.4 \pm 2.0$ & $33.4 \pm 2.7$ & $70.1 \pm 1.9$ & $49.8 \pm 2.0$ \\
\hline
\end{tabular}

${ }^{a}$ Average of three replicates. ${ }^{b}$ The commercial bactericides (thiodiazole-copper and bismerthiazol) were used for comparison of antibacterial activity.

表 2 目标化合物 $2 \mathrm{~b}, 2 \mathrm{e}, 2 \mathrm{f}, 2 \mathrm{k}$ 和 $2 \mathrm{~m}$ 对水稻白叶枯病菌的抑 制率 ${ }^{a}$

Table 2 Inhibitory effects of compounds $2 \mathbf{b}, \mathbf{2 e}, \mathbf{2 f}, \mathbf{2 k}$ and $2 \mathbf{m}$ against Xanthomonas oryzae pv. Oryzae

\begin{tabular}{cccc}
\hline Compd. & Toxic regression equation & $r$ & $\mathrm{EC}_{50} /\left(\mu \mathrm{g} \bullet \mathrm{mL}^{-1}\right)$ \\
\hline $\mathbf{2 b}$ & $y=1.8801+2.2043 x$ & 0.9903 & 26.03 \\
$\mathbf{2 e}$ & $y=2.6641+1.2580 x$ & 0.9927 & 65.73 \\
$\mathbf{2 f}$ & $y=2.6020+1.7664 x$ & 0.9694 & 22.78 \\
$\mathbf{2 k}$ & $y=2.4165+1.6059 x$ & 0.9975 & 40.62 \\
$\mathbf{2 m}$ & $y=1.9605+2.4980 x$ & 0.9969 & 16.47 \\
Bismerthiazol $^{b}$ & $y=1.9605+2.4980 x$ & 0.9800 & 88.51
\end{tabular}

${ }^{a}$ Average of three replicates. ${ }^{b}$ The commercial bactericides agent (bismerthiazol) was used for comparison of antibacterial activity.

浓度下对水稻白叶枯病菌的抑制活性整体要优于取代 基 $\mathrm{R}^{2}$ 为 $4^{\prime}-\mathrm{OCH}_{3}$ 的化合物 $\mathbf{2 a}$ 和 $\mathbf{2 e}$, 其中化合物 $\mathbf{2 b}$ 在 $100 \mu \mathrm{g} / \mathrm{mL}$ 浓度下对水稻白叶枯病菌抑制率达到 $92.8 \%$. 当 $\mathrm{R}^{2}$ 为对氟取代基时，化合物 $\mathbf{2 h}$ 在 100 和 $50 \mu \mathrm{g} / \mathrm{mL}$ 两 个供试浓度下对柑橘溃疡病菌的抑制活性均优于苯环 上其他取代基的化合物, 这可能是氟原子具有较小的体 积及其特殊的生物效应所致.

\section{2 结论}

通过条件探索, 成功设计合成了取代黄酮磷酸二乙 酯目标化合物 14 个; 并进行了抗植物病菌活性测试, 发 现了对水稻白叶枯病菌和相橘溃疡病菌表现出较好活 性的新化合物, 为后续研究奠定了基础.

\section{3 实验部分}

\section{1 仪器与试剂}

${ }^{1} \mathrm{H}$ NMR, ${ }^{13} \mathrm{C}$ NMR, ${ }^{31} \mathrm{P}$ NMR 由 JEOL ECX-500 型
核磁共振仪测定(TMS 为内标或 $85 \%$ 的 $\mathrm{H}_{3} \mathrm{PO}_{4}$ 为外标, $\mathrm{CDCl}_{3}$ 或 DMSO- $d_{6}$ 为溶剂); 质谱由 Agilent $1100 \mathrm{MSD}-$ Trap-VL 质谱仪测定; 高分辨质谱采用美国 Thermofisher 公司 Q Exactive 型液质联用仪; IR 由 Shimadzu Prestige-21 型红外光谱仪测定 ( $\mathrm{KBr}$ 压片); 熔 点用 X-4 数字型熔点仪测定(温度计未经校正). 4-甲氧 基-2-羟基苯乙酮、4-乙氧基-2-羊基苯乙酮和亚磷酸二乙 酯购自上海泰坦科技股份有限公司, 其他试剂均为市售 分析纯或化学纯.

\section{2 中间体及目标化合物的合成}

\subsection{1中间体取代黄酮醇 $1 \mathrm{a} \sim 1 \mathrm{n}$ 的合成方法}

向 $50 \mathrm{~mL}$ 圆底烧瓶中加入取代 2-羟基苯乙酮(1.66 $\mathrm{mmol}) 、$ 取代苯甲醛 $(1.66 \mathrm{mmol})$ 及氢氧化钠 $(3.00 \mathrm{mmol})$, 以乙醇 $(20 \mathrm{~mL})$ 为溶剂, 常温摚拌, 用 TLC 检测至反应 不再发生变化. 继续将反应物冷却到 $0{ }^{\circ} \mathrm{C}$, 加入氢氧化 钠 $(2.00 \mathrm{mmol})$ 和 $30 \%$ 双氧水 $(2.20 \mathrm{mmol})$, 搅拌 $30 \mathrm{~min}$ 后 逐渐升至室温并继续摚拌 12 , 然后 TLC 跟踪反应不再 发生变化. 将反应物倒入冰水中, 在摚拌下加入 6 $\mathrm{mol} / \mathrm{L}$ 的盐酸调节 $\mathrm{pH}$ 值至酸性, 析出大量固体, 滤出固 体, 并用纯净水 $(30 \mathrm{~mL})$ 冲洗 3 次, 用硅胶柱色谱纯化得 到取代黄酮醇 ${ }^{17]}$.

4',7-二甲氧基黄酮醇(1a): 黄色固体，收率 63\%. m.p. 192 194 ${ }^{\circ} \mathrm{C} ;{ }^{1} \mathrm{H}$ NMR $\left(500 \mathrm{MHz}, \mathrm{CDCl}_{3}\right) \delta: 8.23 \sim$ $8.15(\mathrm{~m}, 2 \mathrm{H}), 8.11(\mathrm{~d}, J=8.9 \mathrm{~Hz}, 1 \mathrm{H}), 7.05 \sim 7.01(\mathrm{~m}, 2 \mathrm{H})$, $7.00(\mathrm{~d}, J=0.8 \mathrm{~Hz}, 1 \mathrm{H}), 6.97(\mathrm{dd}, J=8.9,2.3 \mathrm{~Hz}, 1 \mathrm{H})$, $6.93(\mathrm{~d}, J=2.3 \mathrm{~Hz}, 1 \mathrm{H}), 3.92$ (s, $3 \mathrm{H}), 3.88$ (d, $J=2.7 \mathrm{~Hz}$, $3 \mathrm{H}$ ); HRMS calcd for $\mathrm{C}_{17} \mathrm{H}_{15} \mathrm{O}_{5}[\mathrm{M}+\mathrm{H}]^{+}$299.09140, 
found 299.09103.

2',7-二甲氧基黄酮醇(1b): 黄色固体，收率 58\%. m.p. $204 \sim 206{ }^{\circ} \mathrm{C} ;{ }^{1} \mathrm{H}$ NMR $\left(500 \mathrm{MHz}, \mathrm{CDCl}_{3}\right) \delta$ : 8.16 $(\mathrm{d}, J=9.0 \mathrm{~Hz}, 1 \mathrm{H}), 7.57(\mathrm{dd}, J=7.6,1.6 \mathrm{~Hz}, 1 \mathrm{H}), 7.52 \sim$ $7.40(\mathrm{~m}, 1 \mathrm{H}), 7.10(\mathrm{td}, J=7.5,0.7 \mathrm{~Hz}, 1 \mathrm{H}), 7.06(\mathrm{~d}, J=8.3$ $\mathrm{Hz}, 1 \mathrm{H}), 6.99$ (dd, $J=9.0,2.3 \mathrm{~Hz}, 1 \mathrm{H}), 6.88$ (d, $J=2.3 \mathrm{~Hz}$, 1H), 6.47 (s, 1H), 3.89 (s, 3H), 3.87 (s, 3H); HRMS calcd for $\mathrm{C}_{16} \mathrm{H}_{12} \mathrm{O}_{4} \mathrm{Cl}[\mathrm{M}+\mathrm{H}]^{+}$303.04186, found 303.04153.

4'-氯-7-甲氧基黄酮醇 $(\mathbf{1 c})$ : 黄色固体, 收率 71\%。 m.p. 174 $176{ }^{\circ} \mathrm{C} ;{ }^{1} \mathrm{H}$ NMR $\left(500 \mathrm{MHz}, \mathrm{CDCl}_{3}\right) \delta: 8.23 \sim$ $8.15(\mathrm{~m}, 2 \mathrm{H}), 8.13(\mathrm{~d}, J=8.9 \mathrm{~Hz}, 1 \mathrm{H}), 7.55 \sim 7.42(\mathrm{~m}$, $2 \mathrm{H}), 7.23 \sim 7.05(\mathrm{~m}, 1 \mathrm{H}), 7.00(\mathrm{dd}, J=8.9,2.3 \mathrm{~Hz}, 1 \mathrm{H})$, $6.94(\mathrm{~d}, J=2.3 \mathrm{~Hz}, 1 \mathrm{H}), 3.94$ (s, 3H); HRMS calcd for $\mathrm{C}_{16} \mathrm{H}_{12} \mathrm{O}_{4} \mathrm{Cl}[\mathrm{M}+\mathrm{H}]^{+}$303.04186, found 303.04153.

4'-叔丁基-7-甲氧基黄酮醇(1d): 黄色固体, 收率 40\%. m.p. 192 $194{ }^{\circ} \mathrm{C} ;{ }^{1} \mathrm{H}$ NMR (500 MHz, DMSO- $d_{6}$ ) $\delta: 9.36(\mathrm{~s}, 1 \mathrm{H}), 8.23 \sim 8.01(\mathrm{~m}, 2 \mathrm{H}), 7.97(\mathrm{~d}, J=8.9 \mathrm{~Hz}$, 1H), $7.68 \sim 7.42(\mathrm{~m}, 2 \mathrm{H}), 7.23(\mathrm{~d}, J=2.4 \mathrm{~Hz}, 1 \mathrm{H}), 7.02$ (dd, $J=8.9,2.4 \mathrm{~Hz}, 1 \mathrm{H}), 3.89$ (s, 3H), 1.30 (s, 9H); HRMS calcd for $\mathrm{C}_{20} \mathrm{H}_{21} \mathrm{O}_{4}[\mathrm{M}+\mathrm{H}]{ }^{+}$325.14344, found 325.14282 .

4'- 甲氧基-7-乙氧基黄酮醇(1e): 黄色固体, 收率 $60 \%$. m.p. 162 164 ${ }^{\circ} \mathrm{C} ;{ }^{1} \mathrm{H}$ NMR $\left(500 \mathrm{MHz}, \mathrm{CDCl}_{3}\right) \delta$ : 8.20 (d, $J=8.5 \mathrm{~Hz}, 2 \mathrm{H}), 8.11$ (d, $J=8.9 \mathrm{~Hz}, 1 \mathrm{H}), 7.04$ (d, $J=8.4 \mathrm{~Hz}, 2 \mathrm{H}), 6.97$ (d, $J=8.7 \mathrm{~Hz}, 2 \mathrm{H}), 6.92$ (s, 1H), 4.15 (d, $J=6.8 \mathrm{~Hz}, 2 \mathrm{H}), 3.89$ (s, 3H), 1.49 (t, $J=6.7 \mathrm{~Hz}, 3 \mathrm{H})$; HRMS calcd for $\mathrm{C}_{18} \mathrm{H}_{17} \mathrm{O}_{5}[\mathrm{M}+\mathrm{H}]^{+}$313.10705, found 313.10651

2'-甲氧基-7-乙氧基黄酮醇(1f): 黄色固体, 收率 53\%. m.p. 171 $172{ }^{\circ} \mathrm{C}$; ${ }^{1} \mathrm{H}$ NMR (500 MHz, $\left.\mathrm{CDCl}_{3}\right) \delta$ : $8.15(\mathrm{~d}, J=8.9 \mathrm{~Hz}, 1 \mathrm{H}), 7.56(\mathrm{~d}, J=7.6 \mathrm{~Hz}, 1 \mathrm{H}), 7.52 \sim$ $7.43(\mathrm{~m}, 1 \mathrm{H}), 7.10$ (t, $J=7.5 \mathrm{~Hz}, 1 \mathrm{H}), 7.06(\mathrm{~d}, J=8.4 \mathrm{~Hz}$, $1 \mathrm{H}), 7.00 \sim 6.94(\mathrm{~m}, 1 \mathrm{H}), 6.86(\mathrm{~d}, J=2.2 \mathrm{~Hz}, 1 \mathrm{H}), 6.43(\mathrm{~s}$, $1 \mathrm{H}), 4.11$ (q, $J=7.0 \mathrm{~Hz}, 2 \mathrm{H}), 3.88(\mathrm{~s}, 3 \mathrm{H}), 1.46(\mathrm{t}, J=7.0$ $\mathrm{Hz}, 3 \mathrm{H}$ ); HRMS calcd for $\mathrm{C}_{18} \mathrm{H}_{17} \mathrm{O}_{5}[\mathrm{M}+\mathrm{H}]^{+} 313.10705$, found 313.10645 .

2'-溴-7-乙氧基黄酮醇 $(\mathbf{1 g})$ : 黄色固体, 收率 $63 \%$. m.p. $149 \sim 151{ }^{\circ} \mathrm{C} ;{ }^{1} \mathrm{H}$ NMR $\left(500 \mathrm{MHz}, \mathrm{CDCl}_{3}\right) \delta: 8.21$ $(\mathrm{d}, J=15.4 \mathrm{~Hz}, 1 \mathrm{H}), 7.79$ (d, $J=8.8 \mathrm{~Hz}, 1 \mathrm{H}), 7.72$ (dd, $J=$ $7.8,1.6 \mathrm{~Hz}, 1 \mathrm{H}), 7.64$ (dd, $J=8.0,1.1 \mathrm{~Hz}, 1 \mathrm{H}), 7.50$ (d, $J=15.4 \mathrm{~Hz}, 1 \mathrm{H}), 7.41 \sim 7.31(\mathrm{~m}, 1 \mathrm{H}), 7.28 \sim 7.22(\mathrm{~m}, 1 \mathrm{H})$, 6.45 (t, $J=2.4 \mathrm{~Hz}, 1 \mathrm{H}), 4.09$ (q, $J=7.0 \mathrm{~Hz}, 2 \mathrm{H}), 1.44$ (t, $J=7.0 \mathrm{~Hz}, 3 \mathrm{H}$ ); HRMS calcd for $\mathrm{C}_{17} \mathrm{H}_{14} \mathrm{O}_{4} \mathrm{Br}[\mathrm{M}+\mathrm{H}]^{+}$ 361.00700 , found 361.00659 .

4'-氟-7-乙氧基黄酮醇 $(\mathbf{1 h})$ : 黄色固体, 收率 71\%. m.p. $162 \sim 164{ }^{\circ} \mathrm{C} ;{ }^{1} \mathrm{H}$ NMR $\left(500 \mathrm{MHz}, \mathrm{CDCl}_{3}\right) \delta: 8.23$ $(\mathrm{dd}, J=8.7,5.4 \mathrm{~Hz}, 2 \mathrm{H}), 8.11(\mathrm{~d}, J=8.9 \mathrm{~Hz}, 1 \mathrm{H}), 7.20$ (t, $J=8.6 \mathrm{~Hz}, 2 \mathrm{H}), 7.11(\mathrm{~s}, 1 \mathrm{H}), 6.97(\mathrm{dd}, J=8.9,2.0 \mathrm{~Hz}$, $1 \mathrm{H}), 6.91(\mathrm{~d}, J=2.0 \mathrm{~Hz}, 1 \mathrm{H}), 4.14$ (q, $J=7.0 \mathrm{~Hz}, 2 \mathrm{H}), 1.49$ $(\mathrm{t}, J=7.0 \mathrm{~Hz}, 3 \mathrm{H})$; HRMS calcd for $\mathrm{C}_{17} \mathrm{H}_{14} \mathrm{O}_{4} \mathrm{~F}[\mathrm{M}+\mathrm{H}]^{+}$ 301.08706 , found 301.08676 .

4'-氯-7-乙氧基黄酮醇(1i): 黄色固体, 收率 75\%. m.p. $170 \sim 172{ }^{\circ} \mathrm{C} ;{ }^{1} \mathrm{H}$ NMR $\left(500 \mathrm{MHz}, \mathrm{DMSO}-d_{6}\right) \delta$ : $9.68(\mathrm{~s}, 1 \mathrm{H}), 8.21(\mathrm{~d}, J=8.5 \mathrm{~Hz}, 2 \mathrm{H}), 7.95(\mathrm{~d}, J=8.9 \mathrm{~Hz}$, $1 \mathrm{H}), 7.60(\mathrm{~d}, J=8.5 \mathrm{~Hz}, 2 \mathrm{H}), 7.23(\mathrm{~s}, 1 \mathrm{H}), 7.07 \sim 6.85(\mathrm{~m}$, $1 \mathrm{H}), 4.16(\mathrm{dd}, J=13.7,6.8 \mathrm{~Hz}, 2 \mathrm{H}), 1.36$ (t, $J=6.8 \mathrm{~Hz}$, $3 \mathrm{H}$ ); HRMS calcd for $\mathrm{C}_{17} \mathrm{H}_{14} \mathrm{O}_{4} \mathrm{Cl}[\mathrm{M}+\mathrm{H}]^{+}$317.05751, found 317.05701 .

3'-溴-7-乙氧基黄酮醇 $(\mathbf{1 j})$ : 黄色固体, 收率 72\%. m.p. $175 \sim 177{ }^{\circ} \mathrm{C} ;{ }^{1} \mathrm{H}$ NMR $\left(500 \mathrm{MHz}, \mathrm{CDCl}_{3}\right) \delta: 8.35(\mathrm{t}$, $J=1.8 \mathrm{~Hz}, 1 \mathrm{H}), 8.25 \sim 8.18(\mathrm{~m}, 1 \mathrm{H}), 8.11(\mathrm{~d}, J=8.9 \mathrm{~Hz}$, $1 \mathrm{H}), 7.57$ (dd, $J=8.0,1.9,1 \mathrm{H}), 7.39(\mathrm{t}, J=8.0 \mathrm{~Hz}, 1 \mathrm{H})$, 7.15 (d, $J=34.2 \mathrm{~Hz}, 1 \mathrm{H}), 6.99$ (dd, $J=8.9,2.3 \mathrm{~Hz}, 1 \mathrm{H})$, $6.94(\mathrm{~d}, J=2.3 \mathrm{~Hz}, 1 \mathrm{H}), 4.16(\mathrm{q}, J=7.0 \mathrm{~Hz}, 2 \mathrm{H}), 1.50$ (t, $J=7.0 \mathrm{~Hz}, 3 \mathrm{H}$ ); HRMS calcd for $\mathrm{C}_{17} \mathrm{H}_{14} \mathrm{O}_{4} \mathrm{Br}[\mathrm{M}+\mathrm{H}]^{+}$ 361.00700 , found 361.00662 .

4'-甲基-7-乙氧基黄酮醇(1k)：黄色固体，收率 51\%. m.p. $174 \sim 176{ }^{\circ} \mathrm{C} ;{ }^{1} \mathrm{H}$ NMR $\left(500 \mathrm{MHz}, \mathrm{CDCl}_{3}\right) \delta: 8.20 \sim$ $8.06(\mathrm{~m}, 3 \mathrm{H}), 7.32$ (d, J=8.1 Hz, 2H), 7.00 (s, 1H), 6.97 $(\mathrm{dd}, J=8.9,1.9 \mathrm{~Hz}, 1 \mathrm{H}), 6.92(\mathrm{~d}, J=2.0 \mathrm{~Hz}, 1 \mathrm{H}), 4.14$ (q, $J=6.9 \mathrm{~Hz}, 2 \mathrm{H}), 2.43$ (s, 3H), 1.49 (t, $J=6.9 \mathrm{~Hz}, 3 \mathrm{H})$; HRMS calcd for $\mathrm{C}_{18} \mathrm{H}_{17} \mathrm{O}_{4}[\mathrm{M}+\mathrm{H}]^{+}$297.11214, found 297.11188.

7-乙氧基黄酮醇(11): 黄色固体, 收率 60\%. m.p. $157 \sim 159{ }^{\circ} \mathrm{C} ;{ }^{1} \mathrm{H}$ NMR $\left(500 \mathrm{MHz}, \mathrm{CDCl}_{3}\right) \delta: 8.28 \sim 8.18$ $(\mathrm{m}, 2 \mathrm{H}), 8.12$ (d, $J=8.9 \mathrm{~Hz}, 1 \mathrm{H}), 7.53$ (t, $J=7.6 \mathrm{~Hz}, 2 \mathrm{H})$, $7.46(\mathrm{~d}, J=7.3 \mathrm{~Hz}, 1 \mathrm{H}), 7.03$ (s, 1H), 6.98 (dd, $J=8.9,2.2$ $\mathrm{Hz}, 1 \mathrm{H}), 6.93$ (d, $J=2.2 \mathrm{~Hz}, 1 \mathrm{H}), 4.15$ (q, $J=7.0 \mathrm{~Hz}, 2 \mathrm{H})$, $1.49(\mathrm{t}, J=7.0 \mathrm{~Hz}, 3 \mathrm{H})$; HRMS calcd for $\mathrm{C}_{17} \mathrm{H}_{15} \mathrm{O}_{4}[\mathrm{M}+$ $\mathrm{H}]^{+}$283.09649, found 283.09622 .

3',4'-二甲氧基-7-乙氧基黄酮醇(1m): 黄色固体, 收 率 47\%. m.p. $165 \sim 167{ }^{\circ} \mathrm{C} ;{ }^{1} \mathrm{H}$ NMR $\left(500 \mathrm{MHz}, \mathrm{CDCl}_{3}\right)$ $\delta: 8.11(\mathrm{~d}, J=8.9 \mathrm{~Hz}, 1 \mathrm{H}), 7.84(\mathrm{dd}, J=8.5,2.0 \mathrm{~Hz}, 1 \mathrm{H})$, $7.81(\mathrm{~d}, J=2.0 \mathrm{~Hz}, 1 \mathrm{H}), 7.01$ (d, $J=8.6 \mathrm{~Hz}, 1 \mathrm{H}), 6.99$ (d, $J=2.3 \mathrm{~Hz}, 1 \mathrm{H}), 6.97$ (d, $J=2.3 \mathrm{~Hz}, 1 \mathrm{H}), 6.92$ (d, $J=2.2$ $\mathrm{Hz}, 1 \mathrm{H}), 4.15$ (t, J=7.0 Hz, 2H), 3.99 (s, 3H), 3.96 (s, 3H), $1.49(\mathrm{t}, J=7.0 \mathrm{~Hz}, 3 \mathrm{H})$; HRMS calcd for $\mathrm{C}_{19} \mathrm{H}_{19} \mathrm{O}_{6}[\mathrm{M}+$ $\mathrm{H}^{+}$343.11761, found 343.11691.

2',4'-二甲氧基-7-乙氧基黄酩醇(1n): 黄色固体，收 率 56\%. m.p. $177 \sim 179{ }^{\circ} \mathrm{C} ;{ }^{1} \mathrm{H}$ NMR $\left(500 \mathrm{MHz}, \mathrm{CDCl}_{3}\right)$ 
$\delta: 8.12(\mathrm{~d}, J=15.5 \mathrm{~Hz}, 1 \mathrm{H}), 7.80(\mathrm{~d}, J=8.8 \mathrm{~Hz}, 1 \mathrm{H}), 7.60$ $(\mathrm{d}, J=15.5 \mathrm{~Hz}, 1 \mathrm{H}), 7.56(\mathrm{~d}, J=8.6 \mathrm{~Hz}, 1 \mathrm{H}), 6.53(\mathrm{dd}, J=$ 8.6, $2.3 \mathrm{~Hz}, 1 \mathrm{H}), 6.47$ (dd, $J=6.0,2.4 \mathrm{~Hz}, 1 \mathrm{H}), 6.44(\mathrm{t}, J=$ $2.3 \mathrm{~Hz}, 1 \mathrm{H}), 4.08$ (q, $J=7.0 \mathrm{~Hz}, 2 \mathrm{H}), 3.91$ (s, 3H), 3.86 (s, $3 \mathrm{H}$ ), 1.43 (t, $J=7.0 \mathrm{~Hz}, 3 \mathrm{H}$ ); HRMS calcd for $\mathrm{C}_{19} \mathrm{H}_{19} \mathrm{O}_{6}$ $[\mathrm{M}+\mathrm{H}]^{+}$343.11707, found 343.11691.

\subsection{2 目标化合物的合成}

在 $50 \mathrm{~mL}$ 单口烧瓶内加入化合物 $\mathbf{1}(1.00 \mathrm{mmol})$ 、四 氯化碳 $(30 \mathrm{~mL})$ 和三乙胺 $(3.00 \mathrm{mmol})$, 室温摚拌 $0.5 \mathrm{~h}$ 使 其溶解, 然后在冰浴条件下滴加含四氯化碳 $(6 \mathrm{~mL})$ 和 $\mathrm{H}-$ 亚磷酸二乙酯 $(4.00 \mathrm{mmol})$ 的混合溶液, 搅拌 $30 \mathrm{~min}$. 氮 气保护下室温摚拌 $24 \mathrm{~h}$ 后 TLC 监测至新点产生及原料 点不再变化, 用乙酸乙酯萃取 $(30 \mathrm{~mL} \times 3)$, 用 $0.5 \mathrm{~mol} / \mathrm{L}$ 盐酸溶液冲洗 $(15 \mathrm{~mL} \times 3)$, 用 $0.5 \%$ 氢氧化钠溶液中和 $(15 \mathrm{~mL} \times 3)$ 和无水硫酸钠干燥后, 减压蒸馏得到粗品, 用硅胶柱层析 $[V$ (石油醚) $: V($ 乙酸乙酯 $)=1: 1]$ 得到化 合物 2.

$O, O^{\prime}$-二乙基- $O^{\prime \prime}$-(4',7-二甲氧基黄酮-3-基)磷酸酯 (2a): 黄色固体, 收率 $88 \%$. m.p. $77 \sim 79{ }^{\circ} \mathrm{C} ;{ }^{1} \mathrm{H}$ NMR (500 MHz, DMSO- $\left.d_{6}\right) \delta: 7.96(\mathrm{~d}, J=8.8 \mathrm{~Hz}, 1 \mathrm{H}, \mathrm{ArH})$, 7.90 (s, 1H, ArH), 7.89 (s, 1H, ArH), 7.24 (s, 1H, ArH), 7.13 (s, 1H, ArH), 7.11 (s, 1H, ArH), 7.07 (d, $J=10.7 \mathrm{~Hz}$, $1 \mathrm{H}, \mathrm{ArH}), 4.11 \sim 3.93\left[\mathrm{~m}, 4 \mathrm{H}, \mathrm{P}\left(\mathrm{OCH}_{2} \mathrm{CH}_{3}\right)_{2}\right], 3.88(\mathrm{~s}, 3 \mathrm{H}$, $\left.\mathrm{ArOCH}_{3}\right), 3.82\left(\mathrm{~s}, 3 \mathrm{H}, \mathrm{ArOCH}_{3}\right), 1.18 \sim 1.07[\mathrm{~m}, 6 \mathrm{H}$, $\left.\mathrm{P}\left(\mathrm{OCH}_{2} \mathrm{CH}_{3}\right)_{2}\right] ;{ }^{13} \mathrm{C}$ NMR $\left(125 \mathrm{MHz}\right.$, DMSO- $\left.d_{6}\right) \delta$ : $171.59,164.60,162.04,157.10,155.46,133.54,131.01$, $126.97,122.15,117.19,115.61,114.61,101.23,64.76$, 64.71, 56.73, 56.06, 16.38, 16.32; ${ }^{31} \mathrm{P}$ NMR (202 MHz, DMSO- $\left.d_{6}\right) \delta$ : -4.37 ; IR (KBr) $v: 2983,1638,1515,1444$, 1256, 1201, 1016, $845 \mathrm{~cm}^{-1}$; ESI-MS $m / z: 435.2$ ([M+ $\left.\mathrm{H}]^{+}\right), 457.1\left([\mathrm{M}+\mathrm{Na}]^{+}\right)$; HRMS calcd for $\mathrm{C}_{21} \mathrm{H}_{24} \mathrm{O}_{8} \mathrm{P}$ $[\mathrm{M}+\mathrm{H}]^{+}$435.12033, found 435.11966 .

$O, O^{\prime}$-二乙基- $O^{\prime \prime}$-(2',7-二甲氧基黄酮-3-基)磷酸酯 (2b): 黄色油状, 收率 $74 \%$; ${ }^{1} \mathrm{H}$ NMR $(500 \mathrm{MHz}$, DMSO- $\left.d_{6}\right) \delta: 8.00(\mathrm{~d}, J=9.0 \mathrm{~Hz}, 1 \mathrm{H}, \mathrm{ArH}), 7.56$ (dd, $J=$ 9.3, 8.3 Hz, 1H, ArH), 7.48 (dd, J=7.5, 1.6 Hz, 1H, ArH), $7.20(\mathrm{~d}, J=8.4 \mathrm{~Hz}, 1 \mathrm{H}, \mathrm{ArH}), 7.18(\mathrm{~d}, J=2.3 \mathrm{~Hz}, 1 \mathrm{H}$, ArH), $7.11(\mathrm{~d}, J=2.2 \mathrm{~Hz}, 1 \mathrm{H}, \mathrm{ArH}), 7.10 \sim 7.07(\mathrm{~m}, 1 \mathrm{H}$, $\mathrm{ArH}), \quad 3.93 \sim 3.69\left[\mathrm{~m}, 10 \mathrm{H}, \mathrm{P}\left(\mathrm{OCH}_{2} \mathrm{CH}_{3}\right)_{2}, \mathrm{ArOCH}_{3}\right.$, $\mathrm{ArOCH}_{3}$ ], 1.04 [dt, $J=7.6,3.8 \mathrm{~Hz}, 6 \mathrm{H}, \mathrm{P}\left(\mathrm{OCH}_{2} \mathrm{CH}_{3}\right)_{2}$ ]; ${ }^{13} \mathrm{C}$ NMR (125 MHz, DMSO- $\left.d_{6}\right) \delta$ : 171.52, 164.71, 157.66, 157.61, 155.66, 135.09, 133.30, 131.33, 127.07, $120.84,118.87,117.48,115.83,112.43,101.20,64.42$, $64.37,56.78,56.41,16.29,16.24 ;{ }^{31} \mathrm{P}$ NMR (202 MHz, DMSO- $\left.d_{6}\right) \delta:-5.08$; IR (KBr) $v: 2982,1627,1492,1445$,
1256, 1207, 1015, $836 \mathrm{~cm}^{-1}$; ESI-MS m/z: 435.2 ([M+ $\left.\mathrm{H}]^{+}\right)$, $457.1\left([\mathrm{M}+\mathrm{Na}]^{+}\right)$; HRMS calcd for $\mathrm{C}_{21} \mathrm{H}_{24} \mathrm{O}_{8} \mathrm{P}$ $[\mathrm{M}+\mathrm{H}]^{+}$435.12033, found 435.11945.

$O, O^{\prime}$-二乙基- $O^{\prime \prime}$-(4'-氯-7-甲氧基黄酮-3-基)磷酸酯 (2c): 黄色固体, 收率 90\%. m.p. 101 103 ${ }^{\circ} \mathrm{C} ;{ }^{1} \mathrm{H}$ NMR $\left(500 \mathrm{MHz}, \mathrm{CDCl}_{3}\right) \delta: 8.11$ (d, $\left.J=8.8 \mathrm{~Hz}, 1 \mathrm{H}, \mathrm{ArH}\right), 7.90$ (s, 1H, ArH), 7.88 (s, 1H, ArH), 7.47 (s, 1H, ArH), 7.45 (s, $1 \mathrm{H}, \mathrm{ArH}), 6.95$ (dd, $J=8.8,2.3 \mathrm{~Hz}, 1 \mathrm{H}, \mathrm{ArH}), 6.85$ (d, $J=$ $2.3 \mathrm{~Hz}, 1 \mathrm{H}, \mathrm{ArH}), 4.28 \sim 4.06\left[\mathrm{~m}, 4 \mathrm{H}, \mathrm{P}\left(\mathrm{OCH}_{2} \mathrm{CH}_{3}\right)_{2}\right], 3.87$ (s, 3H, $\mathrm{ArOCH}_{3}$ ), 1.24 [td, $J=7.1,1.1 \mathrm{~Hz}, 6 \mathrm{H}$, $\left.\mathrm{P}\left(\mathrm{OCH}_{2} \mathrm{CH}_{3}\right)_{2}\right] ;{ }^{13} \mathrm{C}$ NMR $\left(125 \mathrm{MHz}, \mathrm{CDCl}_{3}\right) \delta: 172.14$, $164.50,157.15,154.11,137.24,134.41,130.34,128.80$, $128.72,127.48,117.62,114.93,100.07,64.97,64.92$, $55.98,16.13,16.07 ;{ }^{31} \mathrm{P}$ NMR $\left(202 \mathrm{MHz}, \mathrm{CDCl}_{3}\right) \delta$ : 4.41; IR (KBr) v: 2980, 1615, 1496, 1440, 1250, 1170, 1026, $840 \mathrm{~cm}^{-1}$; ESI-MS m/z: $439.1\left([\mathrm{M}+\mathrm{H}]^{+}\right), 461.1$ $\left([\mathrm{M}+\mathrm{Na}]^{+}\right)$; HRMS calcd for $\mathrm{C}_{20} \mathrm{H}_{21} \mathrm{ClO}_{7} \mathrm{P}[\mathrm{M}+\mathrm{H}]^{+}$ 439.07079, found 439.07016.

$O, O^{\prime}$-二乙基- $O^{\prime \prime}$-(4'-叔丁基-7-甲氧基黄酮-3-基)磷 酸酯(2d): 黄色固体, 收率 $63 \%$. m.p. $61 \sim 63{ }^{\circ} \mathrm{C} ;{ }^{1} \mathrm{H}$ NMR (500 MHz, $\left.\mathrm{CDCl}_{3}\right) \delta: 8.10(\mathrm{~d}, J=8.8 \mathrm{~Hz}, 1 \mathrm{H}, \mathrm{ArH})$, $7.83(\mathrm{~d}, J=8.2 \mathrm{~Hz}, 2 \mathrm{H}, 2 \times \mathrm{ArH}), 7.49(\mathrm{~d}, J=8.2 \mathrm{~Hz}, 2 \mathrm{H}$, $2 \times \mathrm{ArH}), 6.91(\mathrm{~d}, J=8.6 \mathrm{~Hz}, 1 \mathrm{H}, \mathrm{ArH}), 6.83(\mathrm{~s}, 1 \mathrm{H}, \mathrm{ArH})$, 4.07 [dd, $\left.J=7.1,2.6 \mathrm{~Hz}, 4 \mathrm{H}, \mathrm{P}\left(\mathrm{OCH}_{2} \mathrm{CH}_{3}\right)_{2}\right], 3.83$ (s, $3 \mathrm{H}$, $\left.\mathrm{ArOCH}_{3}\right), 1.31\left[\mathrm{~s}, 9 \mathrm{H}, \operatorname{Ar}\left(\mathrm{CH}_{3}\right)_{3}\right], 1.17[\mathrm{t}, J=7.0 \mathrm{~Hz}, 6 \mathrm{H}$, $\left.\mathrm{P}\left(\mathrm{OCH}_{2} \mathrm{CH}_{3}\right)_{2}\right] ;{ }^{13} \mathrm{C}$ NMR $\left(125 \mathrm{MHz}, \mathrm{CDCl}_{3}\right) \delta: 172.25$, $164.32,157.23,155.74,154.58,134.18,134.13,128.81$, $127.38,125.45,117.69,114.68,100.11,64.72,64.67$, 55.91, 35.04, 31.21, 16.07, 16.01; ${ }^{31} \mathrm{P}$ NMR (202 MHz, $\left.\mathrm{CDCl}_{3}\right) \delta:-4.41$; IR (KBr) v: 2961, 1648, 1441, 1259, 1173, 1035, 993, $845 \mathrm{~cm}^{-1}$; ESI-MS $\mathrm{m} / z: 461.2([\mathrm{M}+$ $\left.\mathrm{H}]^{+}\right), 483.2\left([\mathrm{M}+\mathrm{Na}]^{+}\right)$; HRMS calcd for $\mathrm{C}_{24} \mathrm{H}_{30} \mathrm{O}_{7} \mathrm{P}$ $[\mathrm{M}+\mathrm{H}]^{+}$461.17237, found 461.17148.

$O, O^{\prime}$-二乙基- $O^{\prime \prime}$-(4'-甲氧基-7-乙氧基黄酮-3-基)磷 酸酯(2e): 黄色固体, 收率 73\%. m.p. 87 89 ${ }^{\circ} \mathrm{C} ;{ }^{1} \mathrm{H}$ NMR (500 MHz, $\left.\mathrm{CDCl}_{3}\right) \delta: 8.11(\mathrm{~d}, J=8.9 \mathrm{~Hz}, 1 \mathrm{H}, \mathrm{ArH})$, 7.93 (s, 1H, ArH), 7.91 (s, 1H, ArH), 7.00 (s, 1H, ArH), 6.99 (s, 1H, ArH), 6.93 (d, $J=8.9 \mathrm{~Hz}, 1 \mathrm{H}, \mathrm{ArH}), 6.84$ (s, $1 \mathrm{H}, \quad \mathrm{ArH}), \quad 4.25 \sim 4.05\left[\mathrm{~m}, \quad 6 \mathrm{H}, \quad \mathrm{P}\left(\mathrm{OCH}_{2} \mathrm{CH}_{3}\right)_{2}\right.$, $\left.\mathrm{ArOCH}_{2} \mathrm{CH}_{3}\right], 3.86\left(\mathrm{~s}, 3 \mathrm{H}, \mathrm{ArOCH}_{3}\right), 1.45(\mathrm{t}, J=6.9 \mathrm{~Hz}$, $\left.3 \mathrm{H}, \mathrm{ArOCH}_{2} \mathrm{CH}_{3}\right), 1.24\left[\mathrm{t}, J=7.0 \mathrm{~Hz}, 6 \mathrm{H}, \mathrm{P}\left(\mathrm{OCH}_{2} \mathrm{CH}_{3}\right)_{2}\right]$; ${ }^{13} \mathrm{C}$ NMR $\left(125 \mathrm{MHz}, \mathrm{CDCl}_{3}\right) \delta: 172.26,163.66,161.73$, $157.13,155.31,133.78,130.71,127.37,122.57,117.50$, $114.97,113.91,100.49,64.85,64.81,64.35,55.55,16.16$, $16.10,14.65 ;{ }^{31} \mathrm{P}$ NMR $\left(202 \mathrm{MHz}, \mathrm{CDCl}_{3}\right) \delta$ : -4.30 ; IR 
(KBr) v: 2983, 1623, 1443, 1259, 1185, 1032, 968, 843 $\mathrm{cm}^{-1}$; ESI-MS $m / z: 449.2\left([\mathrm{M}+\mathrm{H}]^{+}\right), 471.1\left([\mathrm{M}+\mathrm{Na}]^{+}\right)$; HRMS calcd for $\mathrm{C}_{22} \mathrm{H}_{26} \mathrm{O}_{8} \mathrm{P}[\mathrm{M}+\mathrm{H}]^{+} 449.13598$, found 449.13522 .

$O, O^{\prime}$-二乙基- $O^{\prime \prime}$-(2'-甲氧基-7-乙氧基黄酮-3-基)磷 酸酯(2f): 黄色固体, 收率 $73 \%$. m.p. $87 \sim 89{ }^{\circ} \mathrm{C} ;{ }^{1} \mathrm{H}$ NMR (500 MHz, $\left.\mathrm{CDCl}_{3}\right) \delta: 8.09$ (d, $\left.J=9.0 \mathrm{~Hz}, 1 \mathrm{H}, \mathrm{ArH}\right)$, 7.49 (d, $J=7.5 \mathrm{~Hz}, 1 \mathrm{H}, \mathrm{ArH}), 7.43$ (t, $J=8.8 \mathrm{~Hz}, 1 \mathrm{H}$, ArH), 7.01 (t, $J=7.4 \mathrm{~Hz}, 1 \mathrm{H}, \operatorname{ArH}), 6.96(\mathrm{~d}, J=8.4 \mathrm{~Hz}$, 1H, ArH), 6.90 (dd, $J=8.8,2.2 \mathrm{~Hz}, 1 \mathrm{H}, \mathrm{ArH}), 6.76$ (d, $J=$ $2.1 \mathrm{~Hz}, 1 \mathrm{H}, \mathrm{ArH}), 4.02$ (q, $J=7.0 \mathrm{~Hz}, 2 \mathrm{H}, \mathrm{ArOCH}_{2} \mathrm{CH}_{3}$ ), $3.99 \sim 3.90\left[\mathrm{~m}, 4 \mathrm{H}, \mathrm{P}\left(\mathrm{OCH}_{2} \mathrm{CH}_{3}\right)_{2}\right.$ ], 3.78 (s, $\left.3 \mathrm{H}, \mathrm{ArOCH}_{3}\right)$, 1.37 (t, $J=6.9 \mathrm{~Hz}, 3 \mathrm{H}, \mathrm{ArOCH}_{2} \mathrm{CH}_{3}$ ), 1.12 [td, $J=7.2,0.9$ $\left.\mathrm{Hz}, 6 \mathrm{H}, \mathrm{P}\left(\mathrm{OCH}_{2} \mathrm{CH}_{3}\right)_{2}\right] ;{ }^{13} \mathrm{C}$ NMR $\left(125 \mathrm{MHz}, \mathrm{CDCl}_{3}\right) \delta$ : $172.09,163.56,157.59,157.54,155.23,135.23,132.35$, $131.07,127.18,120.36,119.26,117.75,115.01,111.40$, $100.54,64.41,64.27,64.20,55.84,16.01,15.94,14.53 ;{ }^{31} \mathrm{P}$ NMR (202 MHz, $\left.\mathrm{CDCl}_{3}\right) \delta:-4.10$; IR (KBr) v: 2981, 1614, 1465, 1444, 1248, 1182, 1032, $987 \mathrm{~cm}^{-1}$; ESI-MS $m / z: 449.2\left([\mathrm{M}+\mathrm{H}]^{+}\right), 471.1\left([\mathrm{M}+\mathrm{Na}]^{+}\right)$; HRMS calcd for $\mathrm{C}_{22} \mathrm{H}_{26} \mathrm{O}_{8} \mathrm{P}[\mathrm{M}+\mathrm{H}]^{+}$449.13598, found 449.13498 .

$O, O^{\prime}$-二乙基- $O^{\prime \prime}$-(2'-澳-7-乙氧基黄酮-3-基)磷酸酯 (2g): 黄色固体, 收率 56\%. m.p. 90 92 ${ }^{\circ} \mathrm{C} ;{ }^{1} \mathrm{H}$ NMR $\left(500 \mathrm{MHz}, \mathrm{CDCl}_{3}\right) \delta: 8.16(\mathrm{~d}, J=8.9 \mathrm{~Hz}, 1 \mathrm{H}, \mathrm{ArH}), 7.72$ (d, $J=8.0 \mathrm{~Hz}, 1 \mathrm{H}, \mathrm{ArH}), 7.67(\mathrm{~d}, J=7.5 \mathrm{~Hz}, 1 \mathrm{H}, \mathrm{ArH})$, 7.46 (t, $J=7.5 \mathrm{~Hz}, 1 \mathrm{H}, \mathrm{ArH}), 7.39$ (t, $J=7.7 \mathrm{~Hz}, 1 \mathrm{H}, \mathrm{ArH})$, $6.98(\mathrm{~d}, J=9.1 \mathrm{~Hz}, 1 \mathrm{H}, \mathrm{ArH}), 6.83$ (s, 1H, ArH), 4.10 (q, $\left.J=7.0 \mathrm{~Hz}, 2 \mathrm{H}, \mathrm{ArOCH}_{2} \mathrm{CH}_{3}\right), 4.02[\mathrm{p}, J=7.1 \mathrm{~Hz}, 4 \mathrm{H}$, $\left.\mathrm{P}\left(\mathrm{OCH}_{2} \mathrm{CH}_{3}\right)_{2}\right], 1.45$ (t, $\left.J=7.0 \mathrm{~Hz}, 3 \mathrm{H}, \mathrm{ArOCH}_{2} \mathrm{CH}_{3}\right), 1.18$ $\left[\mathrm{t}, J=7.1 \mathrm{~Hz}, 6 \mathrm{H}, \mathrm{P}\left(\mathrm{OCH}_{2} \mathrm{CH}_{3}\right)_{2}\right] ;{ }^{13} \mathrm{C}$ NMR $(125 \mathrm{MHz}$, $\left.\mathrm{CDCl}_{3}\right) \delta: 172.23,163.90,157.53,155.85,134.99,133.07$, $132.13,132.01,131.63,127.45,127.39,123.24,117.93$, 115.37, 100.63, 64.75, 64.70, 64.39, 16.04, 15.97, 14.62; ${ }^{31} \mathrm{P}$ NMR $\left(202 \mathrm{MHz}, \mathrm{CDCl}_{3}\right) \delta$ : -5.21 ; IR (KBr) v: 2982, 1645, 1446, 1248, 1183, 1034, 986, $836 \mathrm{~cm}^{-1}$; ESI-MS $m / z: 497.1\left([\mathrm{M}+\mathrm{H}]^{+}\right), 521.1\left([\mathrm{M}+\mathrm{Na}]^{+}\right)$; HRMS calcd for $\mathrm{C}_{21} \mathrm{H}_{23} \mathrm{BrO}_{7} \mathrm{P}[\mathrm{M}+\mathrm{H}]^{+}$497.03593, found 497.03464.

$O, O^{\prime}$-二乙基- $O^{\prime \prime}$-(4'-氟-7-乙氧基黄酮-3-基)磷酸酯 (2h): 黄色固体, 收率 90\%. m.p. $74 \sim 76{ }^{\circ} \mathrm{C} ;{ }^{1} \mathrm{H}$ NMR $\left(500 \mathrm{MHz}, \mathrm{DMSO}-d_{6}\right) \delta: 7.97(\mathrm{dd}, J=8.7,5.8 \mathrm{~Hz}, 3 \mathrm{H}, 3 \times$ ArH), 7.44 (t, $J=8.8 \mathrm{~Hz}, 2 \mathrm{H}, 2 \times \mathrm{ArH}$ ), 7.23 (d, $J=2.1$ $\mathrm{Hz}, 1 \mathrm{H}, \mathrm{ArH}), 7.07$ (dd, $J=8.9,2.1 \mathrm{~Hz}, 1 \mathrm{H}, \operatorname{ArH}), 4.16$ (q, $\left.J=6.9 \mathrm{~Hz}, 2 \mathrm{H}, \mathrm{ArOCH}_{2} \mathrm{CH}_{3}\right), 4.08 \sim 3.90[\mathrm{~m}, 4 \mathrm{H}$, $\left.\mathrm{P}\left(\mathrm{OCH}_{2} \mathrm{CH}_{3}\right)_{2}\right], 1.35$ (t, $\left.J=6.9 \mathrm{~Hz}, 3 \mathrm{H}, \mathrm{ArOCH}_{2} \mathrm{CH}_{3}\right), 1.11$ $\left[\mathrm{t}, J=7.0 \mathrm{~Hz}, 6 \mathrm{H}, \mathrm{P}\left(\mathrm{OCH}_{2} \mathrm{CH}_{3}\right)_{2}\right] ;{ }^{13} \mathrm{C}$ NMR $(125 \mathrm{MHz}$,
DMSO- $\left.d_{6}\right) \delta: 171.64,165.09,163.98,163.10,157.22$, $154.76,133.96,132.03,131.96,127.01,126.63,117.12$, $116.44,116.26,116.08,101.66,64.91,64.82,64.77,16.34$, $16.28,14.87 ;{ }^{31} \mathrm{P}$ NMR $\left(202 \mathrm{MHz}, \mathrm{DMSO}-d_{6}\right) \delta$ : -4.46 ; IR (KBr) v: 2984, 1647, 1507, 1446, 1258, 1187, 1037, $840 \mathrm{~cm}^{-1}$; ESI-MS m/z: $437.1\left([\mathrm{M}+\mathrm{H}]^{+}\right), 459.1([\mathrm{M}+$ $\mathrm{Na}]^{+}$); HRMS calcd for $\mathrm{C}_{21} \mathrm{H}_{23} \mathrm{FO}_{7} \mathrm{P}[\mathrm{M}+\mathrm{H}]^{+}$437.11599, found 437.11502 .

$O, O^{\prime}$-二乙基- $O^{\prime \prime}$-(4'-氯-7-乙氧基黄酮-3-基)磷酸酯 (2i): 黄色固体, 收率 $72 \%$. m.p. $82 \sim 84{ }^{\circ} \mathrm{C} ;{ }^{1} \mathrm{H}$ NMR $\left(500 \mathrm{MHz}, \mathrm{DMSO}-d_{6}\right) \delta: 7.97(\mathrm{~d}, J=8.8 \mathrm{~Hz}, 1 \mathrm{H}, \mathrm{ArH})$, 7.94 (s, 1H, ArH), 7.92 (s, 1H, ArH), 7.67 (s, 1H, ArH), 7.66 (s, 1H, ArH), 7.23 (s, 1H, ArH), 7.07 (d, $J=7.3 \mathrm{~Hz}$, $1 \mathrm{H}, \mathrm{ArH}), 4.17\left(\mathrm{q}, J=6.8 \mathrm{~Hz}, 2 \mathrm{H}, \mathrm{ArOCH}_{2} \mathrm{CH}_{3}\right), 4.09 \sim$ $3.93\left[\mathrm{~m}, 4 \mathrm{H}, \mathrm{P}\left(\mathrm{OCH}_{2} \mathrm{CH}_{3}\right)_{2}\right], 1.35(\mathrm{t}, J=6.9 \mathrm{~Hz}, 3 \mathrm{H}$, $\left.\mathrm{ArOCH}_{2} \mathrm{CH}_{3}\right), 1.11\left[\mathrm{t}, J=7.0 \mathrm{~Hz}, 6 \mathrm{H}, \mathrm{P}\left(\mathrm{OCH}_{2} \mathrm{CH}_{3}\right)_{2}\right] ;{ }^{13} \mathrm{C}$ NMR $\left(125 \mathrm{MHz}, \mathrm{DMSO}-d_{6}\right) \delta: 171.60,164.02,157.21$, $154.45,136.63,134.14,131.11,129.31,128.93,127.00$, 117.11, 116.13, 101.62, 64.92, 64.87, 64.82, 16.32, 16.27, 14.87; ${ }^{31} \mathrm{P}$ NMR (202 MHz, DMSO- $\left.d_{6}\right) \delta$ : -4.53 ; IR (KBr) $v$ : 2982, 1653, 1443, 1260, 1189, 1094, 967, 840 $\mathrm{cm}^{-1}$; ESI-MS $m / z: 453.2\left([\mathrm{M}+\mathrm{H}]^{+}\right), 475.1\left([\mathrm{M}+\mathrm{Na}]^{+}\right)$; HRMS calcd for $\mathrm{C}_{21} \mathrm{H}_{23} \mathrm{ClO}_{7} \mathrm{P}[\mathrm{M}+\mathrm{H}]^{+}$453.08644, found 453.08572 .

$O, O^{\prime}$-二乙基- $O^{\prime \prime}$-( $3^{\prime}$-溴-7-乙氧基黄酮-3-基)磷酸酯 (2j): 黄色固体, 收率 $87 \%$. m.p. $63 \sim 65{ }^{\circ} \mathrm{C} ;{ }^{1} \mathrm{H}$ NMR $\left(500 \mathrm{MHz}, \mathrm{CDCl}_{3}\right) \delta: 8.11$ (d, $\left.J=8.8 \mathrm{~Hz}, 1 \mathrm{H}, \mathrm{ArH}\right), 8.09$ (s, 1H, ArH), 7.88 (d, $J=7.5 \mathrm{~Hz}, 1 \mathrm{H}, \operatorname{ArH}), 7.62$ (d, $J=7.8$ $\mathrm{Hz}, 1 \mathrm{H}, \mathrm{ArH}), 7.37$ (t, $J=7.7 \mathrm{~Hz}, 1 \mathrm{H}, \mathrm{ArH}), 6.95$ (d, $J=$ $8.9 \mathrm{~Hz}, 1 \mathrm{H}, \mathrm{ArH}), 6.86$ (s, 1H, ArH), 4.19 [td, $J=12.8,5.9$ $\left.\mathrm{Hz}, 4 \mathrm{H}, \mathrm{P}\left(\mathrm{OCH}_{2} \mathrm{CH}_{3}\right)_{2}\right], 4.11(\mathrm{dd}, J=13.3,6.5 \mathrm{~Hz}, 2 \mathrm{H}$, $\left.\mathrm{ArOCH}_{2} \mathrm{CH}_{3}\right), 1.45$ (t, $\left.J=6.5 \mathrm{~Hz}, 3 \mathrm{H}, \mathrm{ArOCH}_{2} \mathrm{CH}_{3}\right), 1.26$ $\left[\mathrm{t}, J=7.0 \mathrm{~Hz}, 6 \mathrm{H}, \mathrm{P}\left(\mathrm{OCH}_{2} \mathrm{CH}_{3}\right)_{2}\right] ;{ }^{13} \mathrm{C}$ NMR $(125 \mathrm{MHz}$, $\left.\mathrm{CDCl}_{3}\right) \delta: 172.17,163.95,157.19,153.56,134.68,133.90$, $132.23,131.76,130.06,127.59,127.44,122.51,117.44$, 115.41, 100.46, 65.05, 65.01, 64.46, 16.16, 16.10, 14.62; ${ }^{31} \mathrm{P}$ NMR (202 MHz, $\left.\mathrm{CDCl}_{3}\right) \delta$ : -4.56 ; IR (KBr) v: 2983, 1653, 1445, 1386, 1255, 1204, 1033, $842 \mathrm{~cm}^{-1}$; ESI-MS $m / z: 499.1\left([\mathrm{M}+\mathrm{H}]^{+}\right), 519.1\left([\mathrm{M}+\mathrm{Na}]^{+}\right)$; HRMS calcd for $\mathrm{C}_{21} \mathrm{H}_{23} \mathrm{BrO}_{7} \mathrm{P}[\mathrm{M}+\mathrm{H}]^{+}$497.03593, found 497.03537.

$O, O^{\prime}$-二乙基- $O^{\prime \prime}$-(4'-甲基-7-乙氧基黄酮-3-基)磷酸 酯(2k): 黄色固体, 收率 64\%. m.p. 86 88 ${ }^{\circ} \mathrm{C} ;{ }^{1} \mathrm{H}$ NMR (500 MHz, DMSO- $\left.d_{6}\right) \delta: 7.96(\mathrm{~d}, J=8.3 \mathrm{~Hz}, 1 \mathrm{H}, \mathrm{ArH})$, $7.81(\mathrm{~d}, J=6.4 \mathrm{~Hz}, 2 \mathrm{H}, 2 \times \mathrm{ArH}), 7.38(\mathrm{~d}, J=6.8 \mathrm{~Hz}, 2 \mathrm{H}$, $2 \times \mathrm{ArH}), 7.22(\mathrm{~s}, 1 \mathrm{H}, \mathrm{ArH}), 7.06(\mathrm{~d}, J=7.7 \mathrm{~Hz}, 1 \mathrm{H}, \mathrm{ArH})$, 
$4.16\left(\mathrm{~s}, \quad 2 \mathrm{H}, \quad \mathrm{ArOCH}_{2} \mathrm{CH}_{3}\right), \quad 4.08 \sim 3.88[\mathrm{~m}, 4 \mathrm{H}$, $\left.\mathrm{P}\left(\mathrm{OCH}_{2} \mathrm{CH}_{3}\right)_{2}\right], 2.38$ (s, 3H, $\left.\mathrm{ArCH}_{3}\right), 1.35$ (s, 3H, $\left.\mathrm{ArOCH}_{2} \mathrm{CH}_{3}\right), 1.11\left[\mathrm{t}, J=6.9 \mathrm{~Hz}, 6 \mathrm{H}, \mathrm{P}\left(\mathrm{OCH}_{2} \mathrm{CH}_{3}\right)_{2}\right] ;{ }^{13} \mathrm{C}$ NMR $\left(125 \mathrm{MHz}\right.$, DMSO- $\left.d_{6}\right) \delta: 171.64,163.92,157.19$, $155.59,141.95,133.93,129.69,129.14,127.26,126.98$, 117.11, 115.98, 101.60, 64.89, 64.74, 64.70, 21.62, 16.34, $16.28,14.88 ;{ }^{31} \mathrm{P}$ NMR (202 MHz, DMSO- $d_{6}$ ) $\delta$ : -4.48 ; IR (KBr) v: 2969, 1637, 1608, 1361, 1269, 1227, 1025, $987 \mathrm{~cm}^{-1}$; ESI-MS $m / z: 433.2\left([\mathrm{M}+\mathrm{H}]^{+}\right), 455.1([\mathrm{M}+$ $\mathrm{Na}]^{+}$); HRMS calcd for $\mathrm{C}_{22} \mathrm{H}_{26} \mathrm{O}_{7} \mathrm{P}[\mathrm{M}+\mathrm{H}]^{+}$433.14107, found 433.13983 .

$O, O^{\prime}$-二乙基- $O^{\prime \prime}$-(7-乙氧基黄酮-3-基)磷酸酯（21): 黄色固体, 收率 77\%. m.p. $61 \sim 63{ }^{\circ} \mathrm{C} ;{ }^{1} \mathrm{H}$ NMR (500 $\left.\mathrm{MHz}, \mathrm{CDCl}_{3}\right) \delta: 8.13(\mathrm{~d}, J=8.8 \mathrm{~Hz}, 1 \mathrm{H}, \mathrm{ArH}), 7.96 \sim 7.86$ (m, $2 \mathrm{H}, 2 \times \mathrm{ArH}), 7.54 \sim 7.45(\mathrm{~m}, 3 \mathrm{H}, 3 \times \mathrm{ArH}), 6.95(\mathrm{~d}$, $J=8.9 \mathrm{~Hz}, 1 \mathrm{H}, \mathrm{ArH}), 6.85$ (s, $1 \mathrm{H}, \mathrm{ArH}), 4.22 \sim 3.94[\mathrm{~m}$, $\left.6 \mathrm{H}, \mathrm{P}\left(\mathrm{OCH}_{2} \mathrm{CH}_{3}\right)_{2}, \mathrm{ArOCH}_{2} \mathrm{CH}_{3}\right], 1.45(\mathrm{t}, J=6.9 \mathrm{~Hz}, 3 \mathrm{H}$, $\left.\mathrm{ArOCH}_{2} \mathrm{CH}_{3}\right), 1.21\left[\mathrm{t}, J=7.0 \mathrm{~Hz}, 6 \mathrm{H}, \mathrm{P}\left(\mathrm{OCH}_{2} \mathrm{CH}_{3}\right)_{2}\right] ;{ }^{13} \mathrm{C}$ NMR $\left(125 \mathrm{MHz}, \mathrm{CDCl}_{3}\right) \delta$ : 172.32, 163.79, 157.28, $155.51,134.38,131.00,130.36,129.03,128.50,127.43$, $117.58,115.15,100.53,64.80,64.76,64.38,16.10,16.04$, $14.62 ;{ }^{31} \mathrm{P} \mathrm{NMR}\left(202 \mathrm{MHz}, \mathrm{CDCl}_{3}\right) \delta:-4.45$; IR (KBr) $v$ : 2984, 1627, 1450, 1256, 1188, 1031, 969, $838 \mathrm{~cm}^{-1}$; ESI-MS $m / z$ : $419.2\left([\mathrm{M}+\mathrm{H}]^{+}\right), 441.1\left([\mathrm{M}+\mathrm{Na}]^{+}\right)$; HRMS calcd for $\mathrm{C}_{21} \mathrm{H}_{24} \mathrm{O}_{7} \mathrm{P}[\mathrm{M}+\mathrm{H}]^{+}$419.12542, found 419.12463.

$O, O^{\prime}$-二乙基- $O^{\prime \prime}-\left(3^{\prime}, 4^{\prime}\right.$-二甲氧基-7-乙氧基黄酮-3基)磷酸酯(2m): 黄色固体, 收率 53\%. m.p. $77 \sim 79{ }^{\circ} \mathrm{C}$; ${ }^{1} \mathrm{H}$ NMR $\left(500 \mathrm{MHz}, \mathrm{CDCl}_{3}\right) \delta: 8.13(\mathrm{~d}, J=9.0 \mathrm{~Hz}, 1 \mathrm{H}$, ArH), 7.58 (dd, $J=8.4,2.0 \mathrm{~Hz}, 1 \mathrm{H}, \mathrm{ArH}), 7.53$ (d, $J=2.0$ $\mathrm{Hz}, 1 \mathrm{H}, \mathrm{ArH}), 6.97$ (d, $J=8.5 \mathrm{~Hz}, 1 \mathrm{H}, \mathrm{ArH}), 6.95$ (d, $J=$ $9.0 \mathrm{~Hz}, 1 \mathrm{H}, \mathrm{ArH}), 6.86(\mathrm{~d}, J=2.2 \mathrm{~Hz}, 1 \mathrm{H}, \mathrm{ArH}), 4.25 \sim$ $4.08\left[\mathrm{~m}, 6 \mathrm{H}, \mathrm{P}\left(\mathrm{OCH}_{2} \mathrm{CH}_{3}\right)_{2}, \mathrm{ArOCH}_{2} \mathrm{CH}_{3}\right], 3.95$ (d, $J=6.8$ $\left.\mathrm{Hz}, 6 \mathrm{H}, \mathrm{ArOCH}_{3}, \mathrm{ArOCH}_{3}\right), 1.46$ (t, $J=7.0 \mathrm{~Hz}, 3 \mathrm{H}$, $\left.\mathrm{ArOCH}_{2} \mathrm{CH}_{3}\right), 1.25$ [td, $J=7.1,0.9 \mathrm{~Hz}, 6 \mathrm{H}, \mathrm{P}\left(\mathrm{OCH}_{2}-\right.$ $\left.\mathrm{CH}_{3}\right)_{2}$ ]; ${ }^{13} \mathrm{C} \mathrm{NMR}\left(125 \mathrm{MHz}, \mathrm{CDCl}_{3}\right) \delta: 172.22,163.69$, $157.09,155.18,151.32,148.69,133.87,127.37,122.66$, $122.53,117.46,114.96,111.87,110.70,100.53,64.87$, 64.82, 64.37, 56.17, 56.11, 16.15, 16.09, 14.63; ${ }^{31} \mathrm{P}$ NMR $\left(202 \mathrm{MHz}, \mathrm{CDCl}_{3}\right) \delta:-4.15$; IR (KBr) v: 2982, 1626, $1516,1269,1188,1034,1025 \mathrm{~cm}^{-1}$; ESI-MS $m / z: 479.2$ $\left([\mathrm{M}+\mathrm{H}]^{+}\right), \quad 501.2\left([\mathrm{M}+\mathrm{Na}]^{+}\right)$; HRMS calcd for $\mathrm{C}_{23} \mathrm{H}_{28} \mathrm{O}_{9} \mathrm{P}[\mathrm{M}+\mathrm{H}]^{+}$479.14655, found 479.14590 .

$O, O^{\prime}$-二乙基- $O^{\prime \prime}$-(2',4'-二甲氧基-7-乙氧基黄酮-3基)磷酸酯(2n): 黄色固体, 收率 $66 \%$. m.p. $64 \sim 66{ }^{\circ} \mathrm{C}$;
${ }^{1} \mathrm{H}$ NMR $\left(500 \mathrm{MHz}, \mathrm{CDCl}_{3}\right) \delta: 8.11(\mathrm{~d}, J=9.0 \mathrm{~Hz}, 1 \mathrm{H}$, ArH), 7.45 (d, $J=8.4 \mathrm{~Hz}, 1 \mathrm{H}, \operatorname{ArH}), 6.92$ (dd, $J=9.1,2.3$ $\mathrm{Hz}, 1 \mathrm{H}, \mathrm{ArH}), 6.78$ (d, $J=2.2 \mathrm{~Hz}, 1 \mathrm{H}, \mathrm{ArH}), 6.57$ (dd, $J=$ 8.5, $2.2 \mathrm{~Hz}, 1 \mathrm{H}, \mathrm{ArH}), 6.52(\mathrm{~d}, J=2.2 \mathrm{~Hz}, 1 \mathrm{H}, \mathrm{ArH})$, 4.12 3.97 [m, 6H, P( $\left.\left(\mathrm{OCH}_{2} \mathrm{CH}_{3}\right)_{2}, \mathrm{ArOCH}_{2} \mathrm{CH}_{3}\right], 3.83$ (s, $3 \mathrm{H}, \mathrm{ArOCH}_{3}$ ), 3.79 (s, 3H, $\mathrm{ArOCH}_{3}$ ), 1.41 (t, J=7.0 Hz, $\left.3 \mathrm{H}, \mathrm{ArOCH}_{2} \mathrm{CH}_{3}\right), 1.18$ [td, $J=7.1,0.8 \mathrm{~Hz}, 6 \mathrm{H}$, $\left.\mathrm{P}\left(\mathrm{OCH}_{2} \mathrm{CH}_{3}\right)_{2}\right] ;{ }^{13} \mathrm{C}$ NMR $\left(125 \mathrm{MHz}, \mathrm{CDCl}_{3}\right) \delta: 172.22$, $163.52,163.25,159.07,157.64,155.40,135.32,132.13$, $127.31,117.86,114.91,112.18,104.68,100.59,98.77$, 64.51, 64.47, 64.27, 55.88, 55.66, 16.08, 16.02, $14.61 ;{ }^{31} \mathrm{P}$ NMR (202 MHz, $\left.\mathrm{CDCl}_{3}\right) \delta$ : -4.91 ; IR (KBr) v: 2978, $1648,1284,1250,1172,1027,838 \mathrm{~cm}^{-1}$; ESI-MS $\mathrm{m} / z$ : $479.2\left([\mathrm{M}+\mathrm{H}]^{+}\right), 501.2\left([\mathrm{M}+\mathrm{Na}]^{+}\right)$; HRMS calcd for $\mathrm{C}_{23} \mathrm{H}_{28} \mathrm{O}_{9} \mathrm{P}[\mathrm{M}+\mathrm{H}]^{+} 479.14655$, found 479.14575 .

\section{3 抑菌活性测试}

采用浊度法, 以水稻白叶枯病菌 (Xanthomonas oryzae pv. oryzae) 和柑橘溃疡病菌(Xanthomonas axonopodis pv. citri) 为测试对象, 以商品化药剂噻菌铜 (Thiodiazole copper) 和叶枯唑(Bismerthiazol) 为阳性对照 药剂, 在样品浓度为 100 和 $50 \mu \mathrm{g} / \mathrm{mL}$ 浓度下测试了目 标化合物的离体抑菌活性.

将样品和对照药剂分别配制成浓度为 100 和 50 $\mu \mathrm{g} / \mathrm{mL}$ 的含毒营养肉汤(nutrient broth)液体培养基分别 置于试管中, 测定 $\mathrm{OD}$ 值, 该值为无菌培养基 $\mathrm{OD}$ 值. 然 后接入受试菌种, 在 $28{ }^{\circ} \mathrm{C}, 180 \mathrm{r} / \mathrm{min}$ 恒温摇床振荡培 养 $48 \mathrm{~h}$, 将各个浓度的菌液在分光光度计上测定 OD 值, 该值即为含菌培养基的 OD 值. 按下式计算所测化合物 的抑制率.

抑制率 $=($ 校正后对照培养基菌液 OD 值一校正后含 毒培养基 OD 值)/校正后对照培养基菌液 OD 值 校正 OD 值=含菌培养基 OD 值一无菌培养基 OD 值

辅助材料(Supporting Information) 目标化合物的核 磁共振图谱, ESI-MS 和 HRMS. 这些材料可以免费从本 刊网站(http://sioc-journal.cn/)上下载.

\section{Referenes}

[1] Zhou, J.; Fan, H. T.; Song, B. A.; Jin, L. H.; Pinaki, S. B.; Hu, D. Y. Phosphorus, Sulfur Silicon Relat. Elem. 2010, 186, 81.

[2] Yang, J. Q.; Hu, Y. W.; Gu, Q.; Li, M. G.; Li, M. Q.; Song, B. A. Chin. J. Org. Chem. 2014, 34, 829 (in Chinese). (杨家强, 胡月维, 谷晴, 李明刚, 李明强, 宋宝安, 有机化学, 2014, 34, 829.)

[3] Baird, D. D.; Upchurch, R. P.; Homesley, W. B.; Franz, J. E. Proc. North Cent. Weed Control Conf. 1971, $26,64$.

[4] Hoagland, R. E. Weed Sci. 1980, 393.

[5] Baraldi, P. G.; Beria, I.; Cozzi, P.; Geroni, C.; Espinosa, A.; Gallo, 
M. A.; Romagnoli, R. Bioorg. Med. Chem. 2004, 12, 3911.

[6] Mei, Q. G.; Yuan, W. C.; Wang, C. Chin. J. Org. Chem. 2015, 34, 70 (in Chinese).

(梅青刚, 袁伟成, 王淳, 有机化学, 2015, 35, 70.)

[7] Ruckstuhl, M.; Beretz, A.; Anton, R.; Landry, Y. Biochem. Pharmacol. 1979, 28, 535.

[8] Miles, D. H.; Chittawong, V.; Hedin, P. A.; Kokpol, U. Phytochemistry 1993, 32, 1427

[9] Gu, H. S.; Chen, X.; Zhang, L.; Zhang, J. W.; Li, L. Chin. J. Med. Chem. 2016, 26, 288 (in Chinese).

(谷洪顺, 陈溪, 张兰, 张建伟, 李林, 中国药物化学杂志, 2016, 26, 288.)

[10] Ikemoto, S.; Sugimura, K.; Yoshida, N.; Yasumoto, R.; Wada, S.; Yamamoto, K.; Wadaa, S.; Yamamotoa, K.; Kishimotoa, T. Urology
2000, $55,951$.

[11] Zhang, R. S.; Liu, Y. F.; Chen, Z. Y. Chin. J. Biol. Control 2011, 27, 510 (in Chinese)

(张荣胜, 刘永锋, 陈志谊, 中国生物防治学报, 2011, 27, 510.)

[12] Dong, Y. H.; Wang, L. H.; Xu, J. L.; Zhang, H. B.; Zhang, X. F.; Zhang, L. H. Nature 2001, 411, 813.

[13] Wang, X.; Yin, J.; Shi, L.; Zhang, G.; Song, B. A. Eur. J. Med. Chem. 2014, 77, 65.

[14] Paw, D.; Thomas, R.; Laura, K.; Karina, N.; Thomas, A. M. Int. J. Food Microbiol. 1994, 23, 391

[15] Steinberg, G. M. J. Org. Chem. 1950, 15, 637.

[16] Kenner, G. W.; Williams, N. R. J. Chem. Soc. 1955, 522

[17] Gunduz, S.; Goren, A. C.; Ozturk, T. Org. Lett. 2012, 14, 1576.

(Zhao, C.) 